\title{
Corrigendum to "Prevalence of Stunting among Children Aged 6-23 Months in Kemba Woreda, Southern Ethiopia: A Community Based Cross-Sectional Study"
}

\author{
Eskezyiaw Agedew $\left(\mathbb{D},{ }^{1}\right.$ Tefera Chane $\mathbb{D},{ }^{2}$ and Meaza Demissie ${ }^{3}$ \\ ${ }^{1}$ Department of Public Health, Arba Minch University, Southern Ethiopia, Ethiopia \\ ${ }^{2}$ Department of Public Health, Wolaita Sodo University, 251138 Southern Ethiopia, Ethiopia \\ ${ }^{3}$ Addis Continental Institute of Public Health, Addis Ababa, Ethiopia \\ Correspondence should be addressed to Eskezyiaw Agedew; esklagid@gmail.com
}

Received 10 April 2019; Accepted 11 April 2019; Published 11 June 2019

Copyright (C) 2019 Eskezyiaw Agedew et al. This is an open access article distributed under the Creative Commons Attribution License, which permits unrestricted use, distribution, and reproduction in any medium, provided the original work is properly cited.

In the article titled "Prevalence of Stunting among Children Aged 6-23 Months in Kemba Woreda, Southern Ethiopia: A Community Based Cross-Sectional Study" [1], Dr. Meaza Demissie was missing from the authors' list. Dr. Demissie contributed to the research methodology development, writing, editing, and commenting on the manuscript. The corrected authors' list is shown above.

Also, the authors' contribution section should be updated as follows: "Eskezyiaw Agedew initiated the research, wrote the research proposal, conducted the research, did data entry and analysis, and wrote the paper. Tefera Chane contributed to the designing of methodology and writing of proposal. Meaza Demissie contributed to the research methodology development, writing, editing, and commenting on the manuscript."

\section{References}

[1] E. Agedew and T. Chane, "Prevalence of stunting among children aged 6-23 months in Kemba Woreda, Southern Ethiopia: a community based cross-sectional study," Advances in Public Health, vol. 2015, Article ID 164670, 6 pages, 2015. 


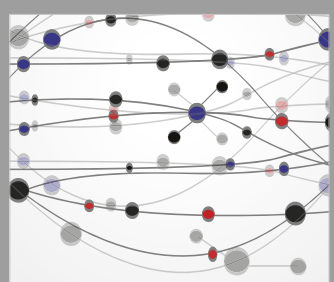

The Scientific World Journal
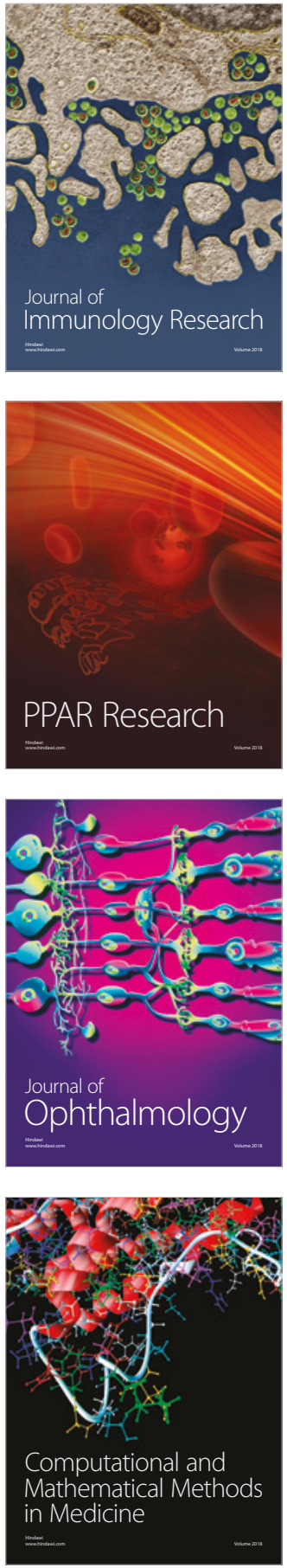

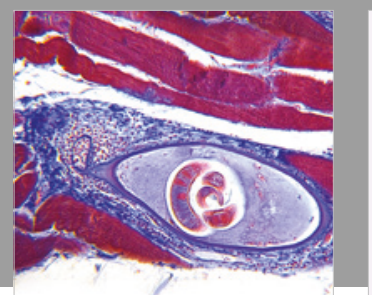

Gastroenterology Research and Practice

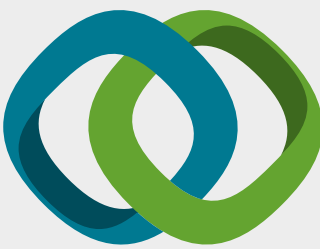

\section{Hindawi}

Submit your manuscripts at

www.hindawi.com
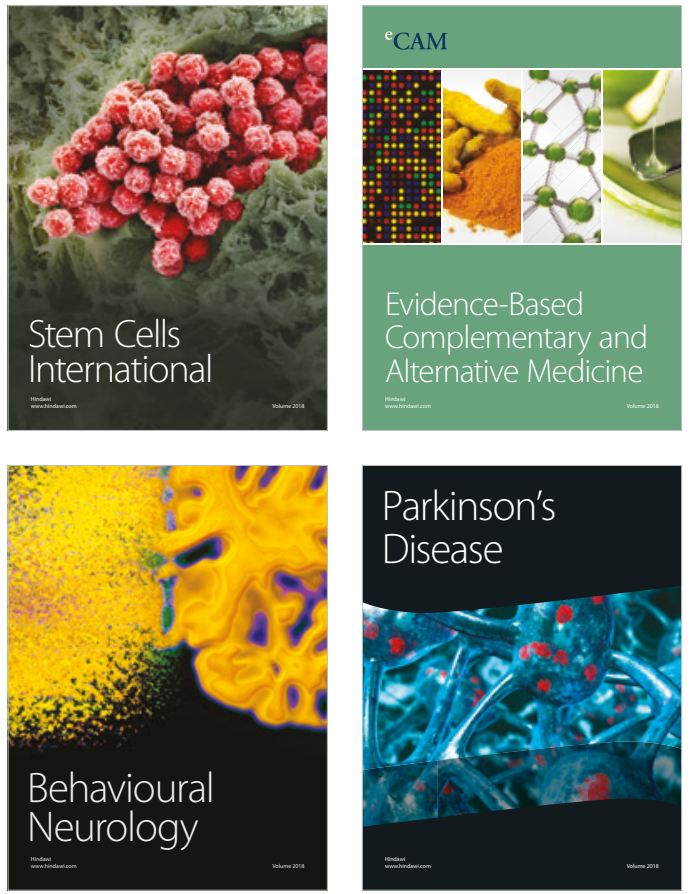

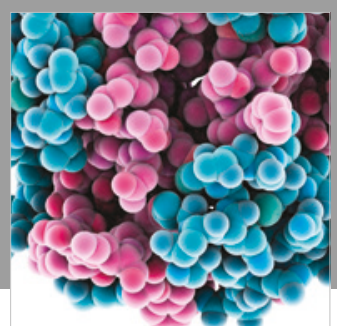

ournal of

Diabetes Research

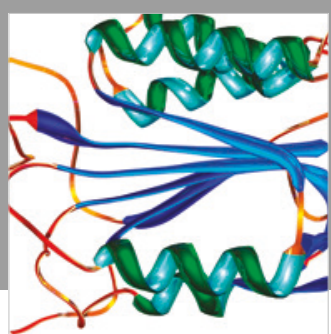

Disease Markers
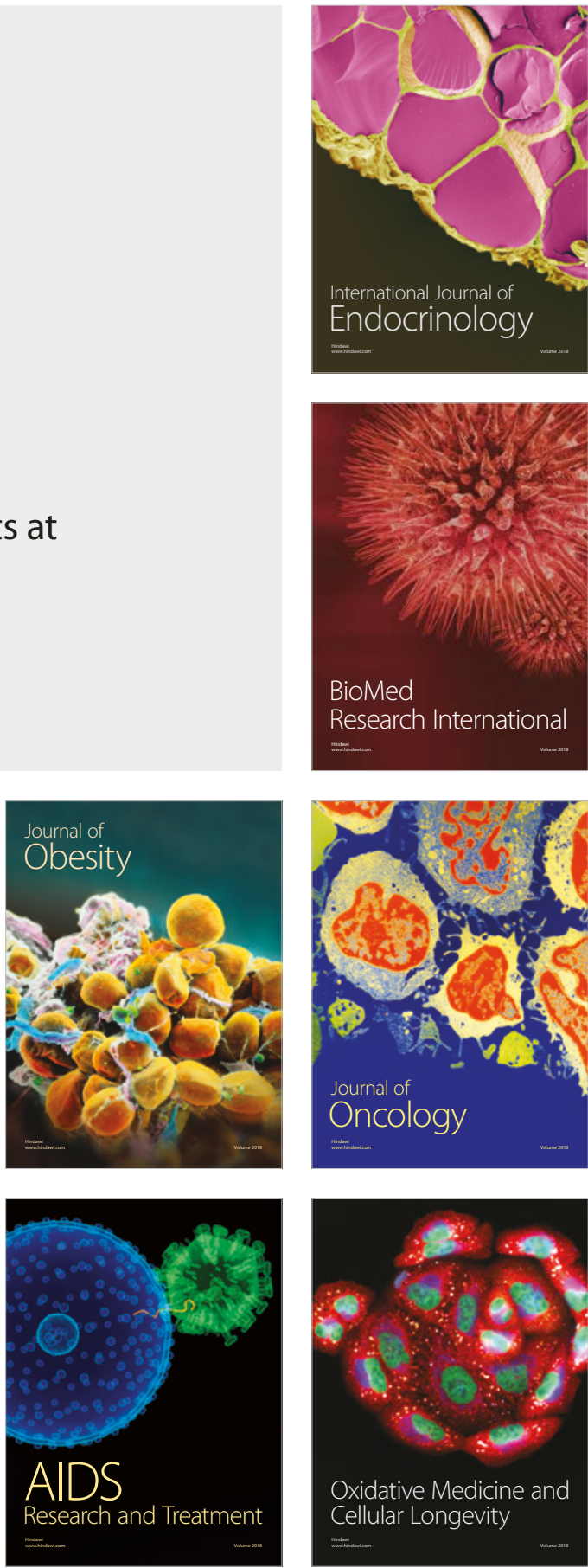